\title{
A large psammomatoid ossifying fibroma with proptosis: A case report
}

\author{
ZHI-YUAN ZHANG* ${ }^{*}$ MEI-PING MIN*, YANG LIU, HONG-QUN JIANG and JIAN ZHANG \\ Department of Otolaryngology Head and Neck Surgery, The First Affiliated Hospital of Nanchang University, \\ Nanchang, Jiangxi 330006, P.R. China
}

Received February 2, 2016; Accepted April 7, 2016

DOI: $10.3892 / \mathrm{mco} .2016 .1104$

\begin{abstract}
The psammomatoid ossifying fibroma (POF) is a rare and benign fibro-osseous lesion predominantly affecting the paranasal sinuses and orbits of children and young adults. The diagnosis and management of the lesion remains challenging. The present study reported a rare case of a large POF in a 39-year-old male patient. The patient had a 30 year history of a slowly growing tumor and this had resulted in right craniofacial deformity, as well as right lateral displacement of the eye ball. Due to the large tumor size, surgical removal of the lesion was the predominant treatment. At 5 months after complete surgical resection, the patient was free from any symptoms. The radiological and histological findings, as well as the surgical management, were presented and the relevant literature was reviewed.
\end{abstract}

\section{Introduction}

As a rare subtype of aggressive ossifying fibroma, psammomatoid ossifying fibroma (POF), also termed juvenile ossifying fibroma, is a benign fibro-osseous lesion predominantly affecting the paranasal sinuses and orbits of children and young adults (1). Histopathology reveals a densely cellular fibrous stroma interspersed with numerous small, spherical ossicles or psammoma bodies. It is locally aggressive, with a high risk of relapse if not completely resected. The present case report presented a large recurrent POF in a male patient.

Correspondence to: Dr Jian Zhang, Department of Otolaryngology Head and Neck Surgery, The First Affiliated Hospital of Nanchang University, 17 Yongwai Zheng Street, Nanchang, Jiangxi 330006, P.R. China

E-mail: jianzhang7@126.com

*Contributed equally

Key words: psammomatoid ossifying fibroma, paranasal sinuses, nasal cavity, proptosis, benign tumors

\section{Case report}

A 39-year-old male presented with a 30 year history of progressive right eye proptosis and decreasing vision without any obvious pain. The tumor had resulted in right craniofacial deformity, as well as right lateral displacement of the eye ball (Fig. 1A and B). Also, the expansile mass had caused partial airway obstruction of the nasal cavity, nasopharynx and oropharynx, accompanying incomplete eyelid closure and restrictive movement of the right eye. Computed tomography (CT) scans revealed a well demarcated, ground-glass opacity expansive lesion extending into the right frontal, maxillary, orbit and paranasal sinuses, with the volume of $9.4 \times 9.8 \times 10.5 \mathrm{~cm}$ (Fig. 1C and D). Due to the large tumor size, surgical removal of the lesion was the predominant treatment (Fig. 2A and B). In addition, pre-operative tracheotomy and external carotid artery embolization was utilized to relieve dyspnea and reduce tumor vascularity. The tumor appeared friable and well-vascularized with an irregular surface. Following the removal of the tumor, the bony anterior skull base remained paper-thin. Gelatin sponge and tela iodoform was placed in the cavity and removed after 6 days.

The histopathology of the specimen revealed gray-white and gray-red soft tissue, and bone fragments were 17x16x6 cm in aggregate (Fig. 2C). Microscopic examination revealed that the tumor with necrotic lesions consisted of dense fibroblastic stroma with interspersed areas of ossification and resembling psammoma bodies (Fig. 3). The pathological diagnosis was POF.

At 13 days after surgery, the patient was discharged without any complications. The patient was free from symptoms and exhibited partial regression of the right eye proptosis. At the 5 month follow-up, satisfactory clinical outcome was observed. The patient recovered well and the right eye-ball mobility was undisturbed. CT scans revealed no recurrence in the area of nasal cavity, nasopharynx and paranasal sinuses.

\section{Discussion}

Benign fibro-osseous craniofacial lesions include fibrous dysplasia, ossifying fibroma and cemento-osseous dysplasia. Ossifying fibroma is divided into conventional ossifying fibroma and juvenile ossifying fibroma. According to the 2005 World Health Organization classification of 

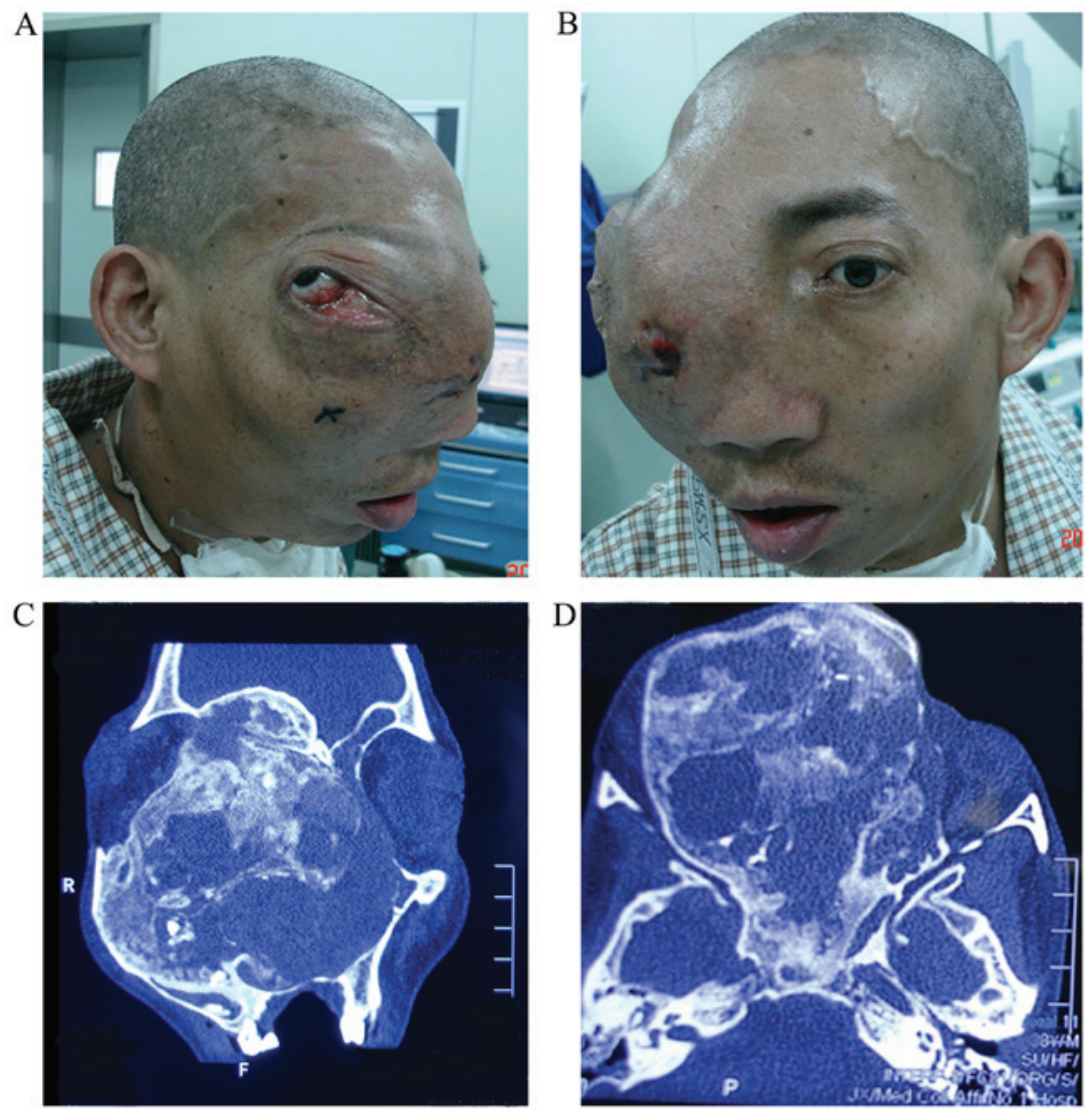

Figure 1. Clinical photographs of the patient. (A) A lateral view of the face showing proptosis and right lateral displacement of the eye ball. (B) A frontal view of the face. (C) Pre-operative CT scans at the coronal position showing a large expansive mass extending into the right orbit and paranasal sinuses. (D) A horizontal position of the head in the pre-operative CT scans. CT, computed tomography.
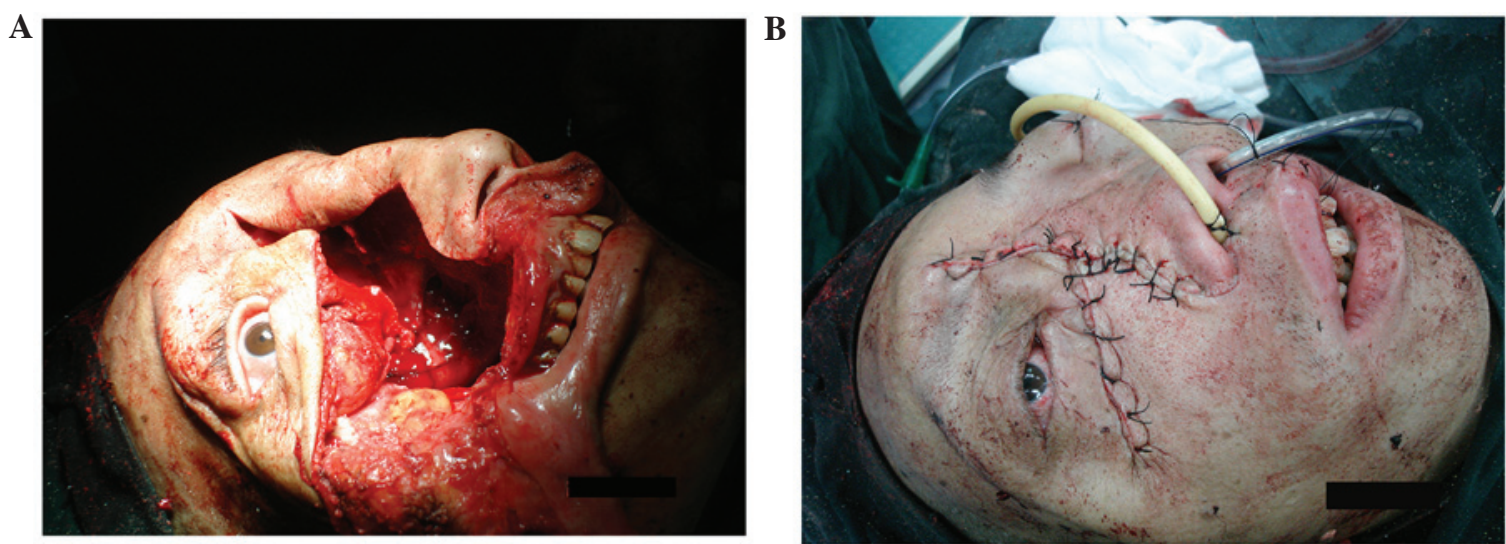

C

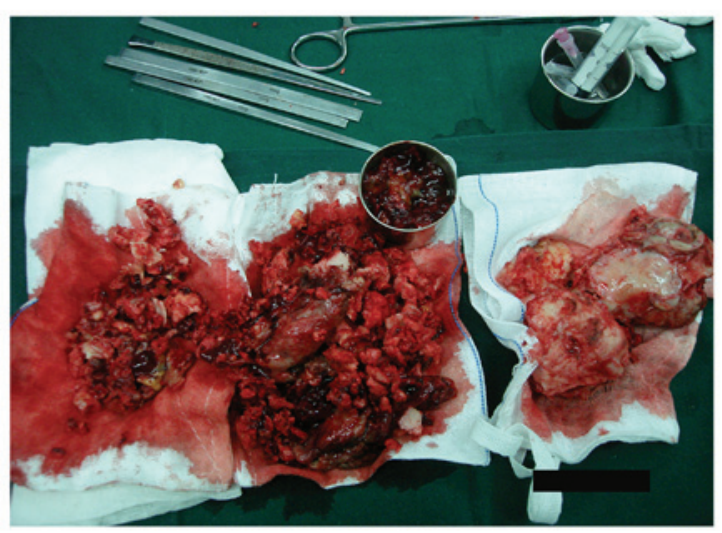

Figure 2. Surgical removal of the lesion. (A) An open surgical path was created and the area of mass excision was clear. (B) The surgical incision suture following the removal of the tumor. (C) The tumor appeared friable and well-vascularized, with an irregular surface. 


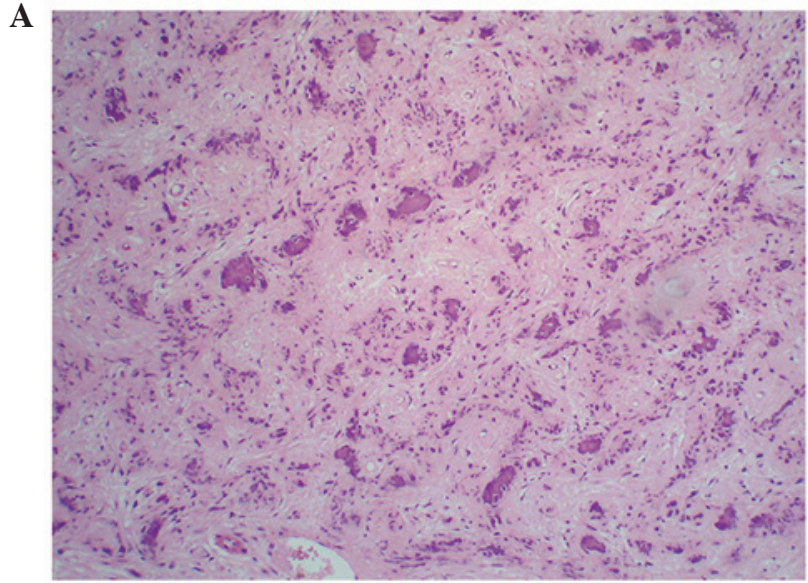

B

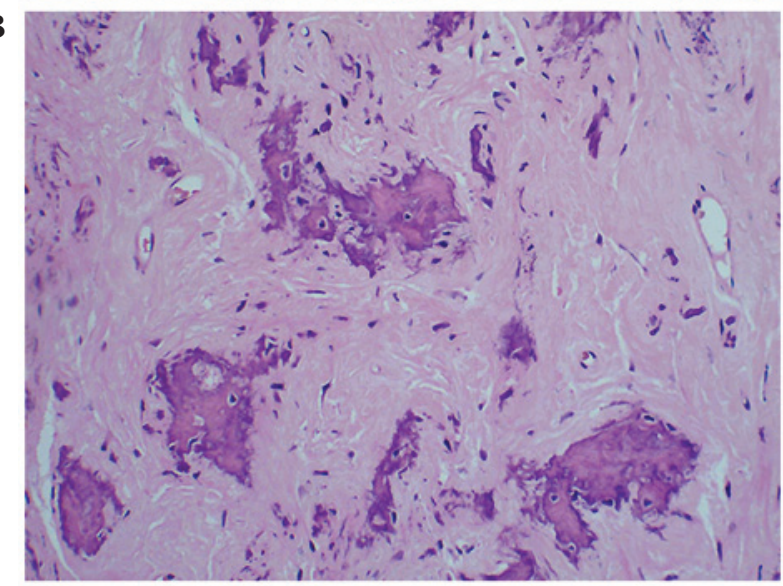

Figure 3. Microscopic images of the psammomatoid ossifying fibroma stained with hematoxylin and eosin. (A) Dense fibroblastic stroma with interspersed areas of ossification, resembling psammoma bodies, was revealed at (A) $\times 100$ and (B) x200 magnification.

odontogenic tumors, juvenile ossifying fibroma is further divided into juvenile POF and juvenile trabecular ossifying fibroma (2). Due to the similarity of certain histopathological features, accurate diagnosis depends on the clinical, radiological and histological features.

POF is a rare subtype of aggressive ossifying fibroma and the etiology of POF remains unclear. POF is distinct from other lesions on the basis of its site, clinical behavior, histopathological features and age of occurrence. POF occurs more commonly in children and young adults, although it has also been reported in patients ranging in ages between 3 months and 72 years (3). POF typically affects regions, including the maxilla bone, paranasal sinuses, orbits and the fronto-ethmoidal complex. By contrast juvenile trabecular ossifying fibroma commonly affects patients younger than those affected by POF, and usually arises in the maxilla or mandible with rapid growth (4).

Clinical manifestations of POF include proptosis, lateral displacement of the eye ball, decreasing vision with progressive blindness and progressive craniofacial deformity. Other symptoms include airway obstruction, headaches, facial swelling and recurrent sinusitis. Local expansion of the lesion may extend into adjacent structures, including the paranasal sinuses, nasal cavity, nasopharynx, palate and cranial cavity.

Radiologically, CT scans of the POF revealed an expansile mass and radiolucent lesion, which is well-circumscribed by a thick bony shell with a multiloculated internal appearance and a content of varying density. Sometimes the lesion has a ground-glass appearance and a variable quantity of internal calcifications, as observed in the present case. In addition, magnetic resonance imaging reveals a mass with a thick outer shell and assists with delineating the extent of the mass. The radiography makes it possible to distinguish POF from other fibro-osseous lesions, including fibrous dysplasia, since fibrous dysplasia is less demarcated and poorly circumscribed by a fibrous capsule (5).

Histologically, the characteristic of POF is the presence of numerous small, spherical ossicles or psammoma bodies that are embedded in a densely cellular fibrous stroma (6). The lesion is well-circumscribed and interspersed with osteoblastic rimming of trabeculae and a large quantity of vascularized fibrous stroma. Certain previous studies have reported concurrent aneurysmal bone cyst formation with POF (7). By contrast, fibrous dysplasia is poorly delineated, with irregular trabeculae of woven bone or ovoid calcifications without lamellar bone and osteoblastic rimming of the trabeculae. Juvenile trabecular ossifying fibroma contains osteoid matrix surrounded by trabeculae of fibrillary osteoid and woven bone.

The predominant treatment for POF is complete surgical excision of the tumor, and partial or incomplete removal leads to recurrence (8). POF is locally aggressive and potentially extends into adjacent vital structures. In the present case, an open surgical approach was employed to resect the tumor as it affords visibility of integrate tumor thereby allowing complete removal. Also, the specimen for pathology is commonly fragmented, making it impossible to evaluate surgical margins appropriately. The successful surgical excision requires multidisciplinary co-operation, involving neurosurgeons, ophthalmologists and otolaryngologists. In the present case, the prognosis of the patient was considered good without malignant degeneration and metastases, which was evidenced by post-operative radiological examination indicating complete removal of the tumor.

\section{References}

1. Linhares P, Pires E, Carvalho B and Vaz R: Juvenile psammomatoid ossifying fibroma of the orbit and paranasal sinuses. A case report. Acta Neurochir (Wien) 153: 1983-1988, 2011.

2. Sarode SC, Sarode GS, Waknis P, Patil A and Jashika M: Juvenile psammomatoid ossifying fibroma: A review. Oral Oncol 47: 1110-1116, 2011.

3. Bohn OL, Kalmar JR, Allen CM, Kirsch C, Williams D and Leon ME: Trabecular and psammomatoid juvenile ossifying fibroma of the skull base mimicking psammomatoid meningioma. Head Neck Pathol 5: 71-75, 2011.

4. Patigaroo SA: Juvenile psammomatoid ossifying fibroma (JPOF) of maxilla-a rare entity. J Maxillofac Oral Surg 10: 155-158, 2011.

5. Figueiredo LM, de Oliveira TF, Paraguassú GM, de Hollanda Valente RO, do Costa WR and Sarmento VA: Psammomatoid juvenile ossifying fibroma: Case study and a review. Oral Maxillofac Surg 18: 87-93, 2014.

6. Zawadzka-Glos L, Brozek-Madry E, Chmielik M, Brzewski M, Biejat A and Maldyk J: Aggressive psammomatoid ossifying fibroma in a 3-month-old boy-A case report. International Journal of Pediatric Otorhinolaryngology Extra 6: 143-145, 2011.

7. Nasser MJ: Psammomatoid ossifying fibroma with secondary aneurysmal bone cyst of frontal sinus. Child Nerv Syst 25: 1513-1516, 2009.

8. Noudel R, Chauvet E, Cahn V, Mérol JC, Chays A and Rousseaux P: Transcranial resection of a large sinonasal juvenile psammomatoid ossifying fibroma. Child Nerv Syst 25: 1115-1120, 2009. 